\title{
Mesodynamics in the SARS nucleocapsid measured by NMR field cycling
}

\author{
Michael W. Clarkson · Ming Lei · Elan Z. Eisenmesser • \\ Wladimir Labeikovsky • Alfred Redfield • \\ Dorothee Kern
}

Received: 27 May 2009/Accepted: 26 June 2009/Published online: 30 July 2009

(c) The Author(s) 2009. This article is published with open access at Springerlink.com

\begin{abstract}
Protein motions on all timescales faster than molecular tumbling are encoded in the spectral density. The dissection of complex protein dynamics is typically performed using relaxation rates determined at high and ultrahigh field. Here we expand this range of the spectral density to low fields through field cycling using the nucleocapsid protein of the SARS coronavirus as a model system. The field-cycling approach enables site-specific measurements of $R_{1}$ at low fields with the sensitivity and resolution of a high-field magnet. These data, together with high-field relaxation and heteronuclear NOE, provide evidence for correlated rigid-body motions of the entire $\beta$-hairpin, and corresponding motions of adjacent loops with a time constant of $0.8 \mathrm{~ns}$ (mesodynamics). MD simulations substantiate these findings and provide direct verification of the time scale and collective nature of these motions.
\end{abstract}

Keywords Dynamics $\cdot$ Field-cycling $\cdot$ Collective motions

\section{Introduction}

The dynamic nature of proteins is one of the key elements of their function. Although this principle is widely accepted,

M. W. Clarkson · M. Lei · E. Z. Eisenmesser ·

W. Labeikovsky $\cdot$ D. Kern $(\bowtie)$

Department of Biochemistry and Howard Hughes Medical Institute, MS009 Brandeis University, Waltham, MA 02452, USA

e-mail: dkern@brandeis.edu

A. Redfield $(\bowtie)$

Department of Biochemistry, MS009 Brandeis University,

Waltham, MA 02452, USA

e-mail: redfield@brandeis.edu the detailed characterization of protein motions at atomic resolution is much more challenging than determining the average static structure. High field NMR is a particularly powerful method for studying protein dynamics at atomic resolution over a wide array of time scales and has therefore extensively been used in the last two decades. The major emphasis on the development of ultra-high-field magnets for an increase in sensitivity and resolution has allowed investigations on ever-larger biomolecular systems. Ironically, this high-field regime is not as sensitive to mesodynamics as relaxation data at lower fields. Low-field $R_{1}$ relaxation experiments covering ${ }^{1} \mathrm{H}$ Larmor frequencies up to several tens of $\mathrm{MHz}$ have been performed using fieldcycling relaxometers (Kimmich 1996; Luchinat and Parigi 2007). Due to the low resolution, these experiments have usually probed the relaxation of solvent protons as reporters, and are therefore limited. Here we report $R_{1}$ relaxation data of a protein at low field, but with atomic resolution, using a field-cycling apparatus in a commercial $500 \mathrm{MHz}$ magnet (Redfield 2003). By physically moving the sample in the magnet bore the relaxation behavior can be investigated at various low fields; however, the return of the sample to its initial position allows these measurements with the resolution and sensitivity of the high field.

This approach has been successfully applied to studies of lipids and DNA, using one-dimensional ${ }^{31} \mathrm{P}$ and ${ }^{13} \mathrm{C}$ spectra (Roberts et al. 2004; Sivanandam et al. 2009). Here we use two-dimensional ${ }^{1} \mathrm{H}^{15} \mathrm{~N}$ heteronuclear experiments spanning from 17.3 to $91.2 \mathrm{MHz}\left({ }^{15} \mathrm{~N}\right.$ Larmor frequency will be used throughout, corresponding to $170-900 \mathrm{MHz}$ "spectrometers") to investigate the dynamics of the N-terminal domain of the SARS (Severe Acute Respiratory Syndrome) nucleocapsid protein (SARSN).

SARSN is a multifunctional protein (Surjit and Lal 2008) implicated in RNA binding (Tan et al. 2006; Zuniga 
et al. 2007), capsid assembly (Hsieh et al. 2005; Saikatendu et al. 2007), cell-cycle disruption (Surjit et al. 2006), and immune response to the SARS virus (Zhang et al. 2007). Previous experiments have indicated a flexible $\beta$-hairpin in addition to a relatively rigid antiparallel $\beta$-sheet based on the $\left\{{ }^{1} \mathrm{H}\right\}-{ }^{15} \mathrm{~N}$ heteronuclear NOE at $500 \mathrm{MHz}$ (Huang et al. 2004). Sampling the spectral density over this wider range enabled us to robustly determine the time scale of motions of the hairpin and additional loops (Fig. 1). Heteronuclear $\left\{{ }^{1} \mathrm{H}\right\}-{ }^{15} \mathrm{~N}$ NOE at differing fields $(50.7-91.2 \mathrm{MHz})$ were particularly useful for establishing the internal correlation times in the disordered regions. Matching internal correlation times of about $0.8 \mathrm{~ns}$ for many of these residues are suggestive of correlated motions, which were buttressed by molecular dynamics simulations in explicit solvent.

\section{Methods and materials}

\section{Relaxation measurements}

High resolution $2 \mathrm{D}{ }^{15} \mathrm{~N} R_{1}$ relaxation measurements at 17.3 and $30.2 \mathrm{MHz}$ were obtained using a Varian INOVA 500 equipped with a Varian indirect probe and a homebuilt field-cycling device (Redfield 2003) to move the sample

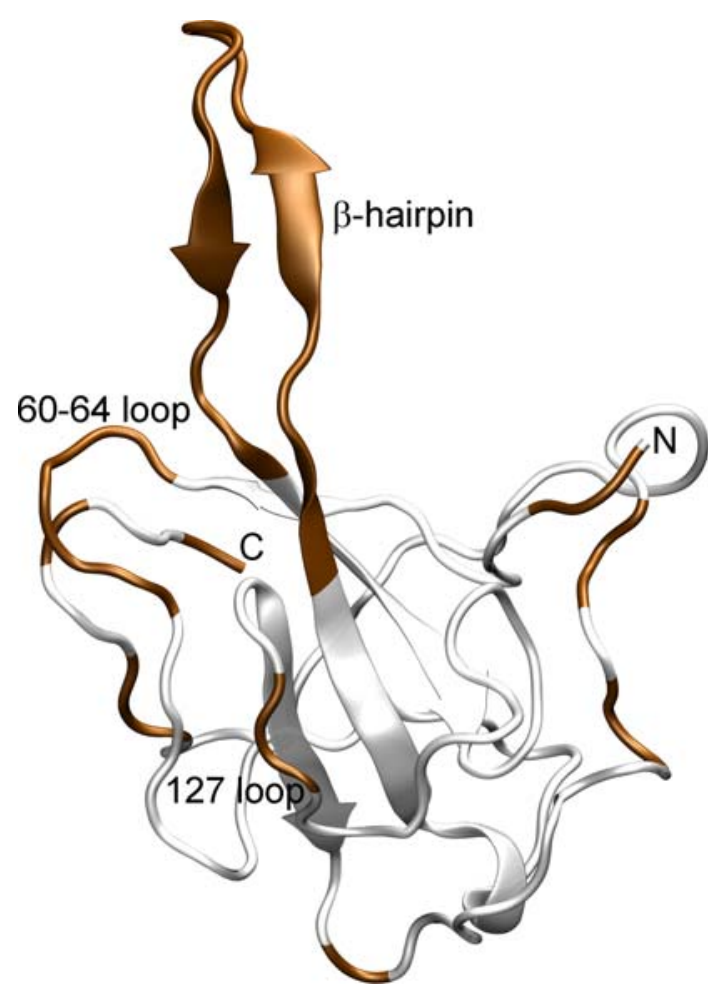

Fig. 1 Regions with mesodynamics (correlated motions with a time constant of about $0.8 \mathrm{~ns}$ ) cluster structurally in SARSN. Residues that are fit to model 5 and internal correlation times $\left(\tau_{\mathrm{s}}\right)$ around $0.8 \mathrm{~ns}$ are shown on the structure of SARSN (PDB code 2OFZ) in dark orange from the center of the magnet to the desired lower fringe fields. A standard thin-walled $5 \mathrm{~mm}$ NMR tube is modified with plastic inserts epoxied above and below the sample to avoid bubbles. Previous iterations of this device used a pneumatic driver for the shuttle, but the violent deceleration of the sample at either end of transit apparently led to protein denaturation, even though samples are tightly sealed and degassed to minimize turbulence as described previously (Redfield 2003). The system has therefore been rebuilt to use a stepper motor to drive a timing belt and push rod to move the sample, allowing for more controlled deceleration (Redfield, in preparation). Experiments with ferro-cytochrome $\mathrm{c}$ were performed to verify that temperature variations during field-cycling could be neglected (data not shown).

Fringe field strengths versus height were measured with magnetometers as described (Redfield 2003) and checked with a commercial Gaussmeter (Lake Shore). The position of the sample in the fringe field was controlled by digital input to the stepper motor. The field strengths reported in the results reflect the measured field at the center of the sample. $R_{1}$ measurements at low field used a standard experiment (Farrow et al. 1994) with the addition of commands to control the field-cycling apparatus, and 100-150 ms delays to accommodate the raising and lowering of the sample. This transit delay was adjusted to account for the greater distance necessary to sample low fields. In order to compensate for signal attenuation due to relaxation in transit, 4-8 times as many transients were acquired for these experiments as at high field. Fieldcycling did not affect the lock to a greater extent than did a strong gradient pulse, and no degradation of the lock was observed over the course of the experiment. Proton inversion pulses during the relaxation period were not feasible because of the inhomogeneity of the fringe field and absence of a transmitter coil at the relevant height.

High-field NMR spectra were acquired on Varian INOVA 500, 600, and $900 \mathrm{MHz}$ spectrometers equipped with triple resonance probes and $z$-axis gradients, as well as a Bruker AVANCE-800 spectrometer equipped with a cryoprobe. $R_{1}, R_{2}$, and NOE data were collected using standard ${ }^{15} \mathrm{~N}$ relaxation experiments (Farrow et al. 1994), in an interleaved manner.

Processing and fitting of relaxation data

Spectra were processed using NMRPipe (Delaglio et al. 1995) software and analyzed using NMRViewJ (Johnson and Blevins 1994). For $R_{1}$ and $R_{2}$, peak intensities were fit to mono-exponential decay equations using an in-house program. Errors in the measurements were assessed from base-plane noise and duplicated points; because of the interleaved acquisition, both approaches resulted in similar 
estimated errors. Model-free fits were performed using relxn2.2 (Lee et al. 1999) and the graphical front-end rvi (Clarkson et al. 2006); only relaxation data from $50.7 \mathrm{MHz}$ and above were used in the final fits. Data were weighted by error for $\chi^{2}$ minimization, which for purposes of fitting was defined as the larger of the fitted error or $5 \%$. Excluding flexible residues (Vuister et al. 1993), a $\tau_{\mathrm{m}}$ of $11.9 \mathrm{~ns}$ was determined by global minimization and used in all subsequent fits. An estimation of tumbling anisotropy using local fits of $\tau_{\mathrm{m}}$ and the 1SSK structure (Huang et al. 2004) with the program qfit (Lee et al. 1999) indicated $\mathrm{D}_{\|} / \mathrm{D}_{\perp}$ of 0.94 , and anisotropy was therefore disregarded in subsequent fitting procedures. Errors in single fits were assessed using a Monte Carlo method in which random values consistent with the measured errors were added to the original data and the fit repeated. The total number of Monte Carlo simulations used was 150 for each residue.

Model selection was performed using the Bayesian information criterion (BIC) with model elimination (d'Auvergne and Gooley 2003) and a modified jackknife approach in which 1-3 relaxation rates or NOEs were eliminated in each attempted fit. Residues for which more than one violation of model-free constraints (order parameters greater than one or less than zero, or $\tau_{\mathrm{e}} / \tau_{\mathrm{s}} \geq \tau_{\mathrm{m}}$ ) occurred during the jackknife procedure were shifted to a simpler model. No residues experienced multiple violations during the jackknife when model 2 was used. The initial rounds of model selection included models that incorporate $R_{\mathrm{EX}}$ corrections to the $R_{2}$ data (models 3 and 4). However, these models were not ultimately selected for any residues because CPMG relaxation dispersion gave no indication of $R_{\text {EX }}$ (data not shown), and refinement of model selection excluded those models. Accordingly, the only models used in the final analysis were model $2\left(S^{2}, \tau_{\mathrm{e}}\right)$ (Lipari and Szabo 1982), and model $5\left(S_{\mathrm{s}}^{2}, S_{\mathrm{f}}^{2}, \tau_{\mathrm{s}}\right)$ (Clore et al. 1990). The final model-free fits excluded the low-field data in the interest of accuracy (see below), but including these data did not generally change the fitted order parameter by more than $5 \%$.

Parameter variability in the jackknife was heterogeneous. The standard deviations of the order parameter $\left(S^{2}\right.$ or $\left.S_{\mathrm{s}}^{2} \times S_{\mathrm{f}}^{2}\right)$ and internal correlation time $\left(\tau_{\mathrm{e}}\right.$ or $\left.\tau_{\mathrm{s}}\right)$ were 4.6 and $96 \%$ of the average value for a residue, respectively. For residues with a narrow $R_{1}$ distribution, these errors were reduced to 2.9 and $11.9 \%$, respectively, suggesting that the internal correlation time in particular is fit much more robustly for these residues.

Molecular dynamics simulation

Solvent molecules and counter-ions were added to the PDB file 2OFZ (Saikatendu et al. 2007) by the program solvate
1.0 (Grubmuller 1995). Atoms beyond $42 \AA$ from the center were deleted; the minimum distance between any protein atom and the spherical surface was $16 \AA$. The stochastic boundary condition (Brooks and Karplus 1983; Brunger et al. 1984) was imposed to prevent solvent from departing from the water sphere. The CHARMM program (Brooks et al. 1983) with the all-atom force field (MacKerell et al. 1998) and CMAP correction (Mackerell et al. 2004) was used for minimization and simulation. The non-bonded interaction cut-off distance was set to be $14 \AA$, and the simulation timestep was 2 fs. Hydrogen atoms were constrained by the SHAKE algorithm. The system was minimized, then heated from 50 to $300 \mathrm{~K}$ in 200 ps. The two production runs were $15 \mathrm{~ns}$ in duration.

\section{Protein expression and purification}

The SARSN construct was a gift from Dr. Hualiang Jiang (Luo et al. 2004). E. Coli BL21 (DE3) cells were grown in ${ }^{15} \mathrm{~N}$ M9 medium to an OD of 0.6 and induced with $1 \mathrm{mM}$ IPTG. Cells were harvested by centrifugation and resuspended in lysis buffer (0.1 M Tris, $0.5 \mathrm{M} \mathrm{NaCl}, \mathrm{pH} 7.0)$ with a protease inhibitor cocktail (Calbiochem). Following lysis by sonication, cellular debris was removed by centrifugation. The lysate was then dialyzed against $50 \mathrm{mM}$ $\mathrm{Na}_{4} \mathrm{H}_{2} \mathrm{PO}_{4}, 50 \mathrm{mM} \mathrm{NaCl} \mathrm{pH} 7.0$ and purified over SP-sepharose equilibrated in the same buffer, eluting with $2 \mathrm{M} \mathrm{NaCl}$. Following a repeat dialysis step, the protein was purified over Q-sepharose using the same buffer system. Following dialysis into the NMR buffer (50 mM HEPES, $150 \mathrm{mM} \mathrm{NaCl}, 0.02 \% \mathrm{NaN}_{3} \mathrm{pH} 7.0$ ), the protein was purified over S-100 sepharose and concentrated to $1 \mathrm{mM}$. NMR samples contained $10 \% \mathrm{D}_{2} \mathrm{O}$. All separation media were purchased from GE Healthcare.

\section{Results and discussion}

Field dependence of $\mathrm{R}_{1}$ relaxation rates

At $500 \mathrm{MHz}(11.7 \mathrm{~T}), R_{1}$ values are roughly constant across the entire SARSN, but $R_{1}$ heterogeneity significantly increased as the magnitude of the field was changed in either direction (Fig. 2). When $R_{1}$ was measured at a 17.3 MHz field sampled by the field-cycling apparatus, for several residues $R_{1}$ was decreased by as much as $25 \%$ relative to the majority of the residues (Fig. 2a). This reduction is also observed at $7 \mathrm{~T}$, but to a lesser extent. These residues were primarily localized to the $\beta$-hairpin (residues 90-108) and a loop region spanning residues 60-64. On the contrary, increasing the field strength above $11.7 \mathrm{~T}$ resulted in an enhanced $R_{1}$ for the same set of residues relative to the rest of the protein. At the highest 

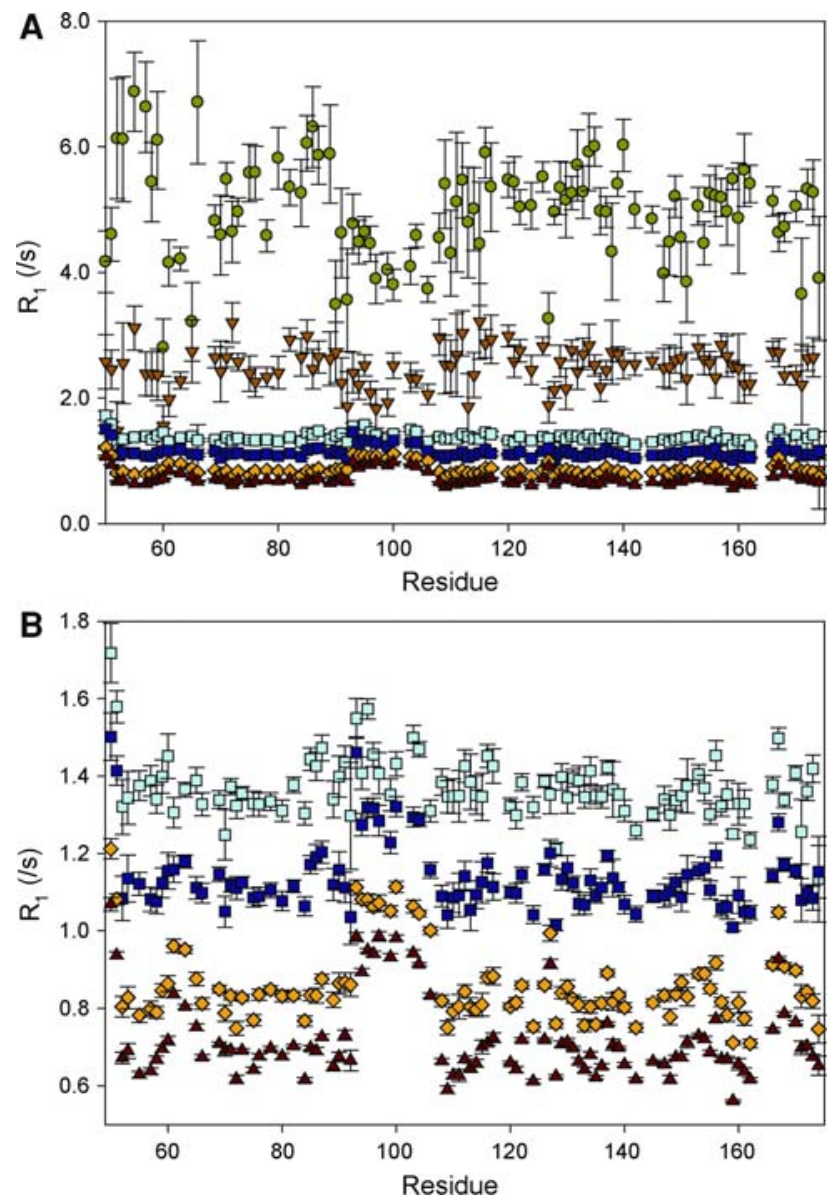

Fig. 2 The field dependence of $R_{1}$ for residues in SARSN. a $R_{1}$ values at $17.3 \mathrm{MHz}$ (green open circle) and $30.2 \mathrm{MHz}$ (orange inverted filled triangle) measured by field-cycling and at $50.7 \mathrm{MHz}$ (light blue open square), $60.8 \mathrm{MHz}$ (dark blue filled square), $81.1 \mathrm{MHz}$ (yellow open diamond), and $91.2 \mathrm{MHz}$ (red filled triangle). $\mathbf{b}$ Expansion of the highfield data. The crossover for the $\beta$-hairpin (residues 90-108)-lower $R_{1}$ values than the stable and rigid region of the protein at low fields and higher values for fields higher than $50.7 \mathrm{MHz}$ - is apparent

field employed in this study, $91.2 \mathrm{MHz}$, the enhancement was as high as $40 \%$ (Fig. 2b).

Qualitatively, this " $R_{1}$ crossover" implies that these residues have greater flexibility than the remainder of the protein. Lower $R_{1}$ values are expected for flexible residues at low field because the internal motion depresses the spectral density in the extreme narrowing limit $\left(\omega \tau_{\mathrm{m}} \ll 1\right)$. On the contrary, in the spin-diffusion limit $\left(\omega \tau_{\mathrm{m}} \gg 1\right)$, flexible residues are expected to have a greater spectral density and thus a higher $R_{1}$ than rigid residues. Moreover, the particular frequency at which the crossover occurs defines an approximate range of internal correlation times for these residues. For a protein of this size, the observed $R_{1}$ crossover point implies a motion with an internal correlation time in the range of $0.5-1.5 \mathrm{~ns}$.

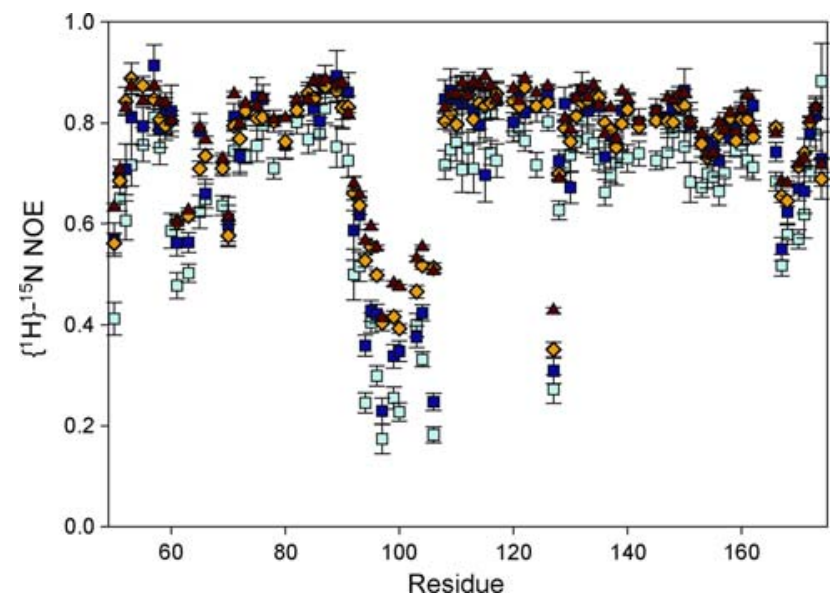

Fig. 3 The field dependence of the steady-state NOE for residues in SARSN. NOE data are shown for $50.7 \mathrm{MHz}$ (light blue open square), $60.8 \mathrm{MHz}$ (dark blue filled square), 81.1 MHz (yellow open diamond), and $91.2 \mathrm{MHz}$ (red filled triangle)

The data collected at low field using field cycling show larger differences in $R_{1}$ between rigid and flexible residues than the high and ultra-high-field data. Uncertainties for the low-field data are larger because relaxation during the 200 $300 \mathrm{~ms}$ transit time and increased $R_{1}$ relaxation rates at lower fields diminishes the intensity of the signals from the protein. Despite these imperfections, the field-cycling data are of sufficient quality to provide valuable insight into the dynamics of this protein at atomic resolution.

Field dependence of the $\left\{{ }^{1} \mathrm{H}\right\}-{ }^{15} \mathrm{~N}$ heteronuclear NOEs

The $\left\{{ }^{1} \mathrm{H}\right\}-{ }^{15} \mathrm{~N}$ heteronuclear NOEs for the $\beta$-hairpin also display a pronounced field-dependence between 50.7 and 91.2 $\mathrm{MHz}$ (Fig. 3) with a confirmation of the previously reported strong NOEs at $50.7 \mathrm{MHz}$ (Huang et al. 2004). The attenuation of the NOEs at higher fields strongly suggests an internal mesodynamic fluctuation. The ${ }^{15} \mathrm{~N}$ NOE is the ratio $R_{\mathrm{NOE}} \gamma_{\mathrm{H}} / R_{1} \gamma_{\mathrm{N}}$, where $R_{\mathrm{NOE}}$ is approximately proportional to $J\left(\omega_{\mathrm{H}}\right)$ since $\omega_{\mathrm{N}} \ll \omega_{\mathrm{H}}$, and $R_{1}$ is proportional to $J\left(\omega_{\mathrm{N}}\right)$ (Abragam 1961). This ratio changes little for rigid residues over a twofold change in Larmor frequency because the respective spectral density values are essentially constant over this frequency range. In contrast, the observed field-dependence of the NOE for flexible residues indicates the presence of a transition in the spectral density over this frequency range. Moreover, the range of Larmor frequencies sampled defines upper and lower bounds for the internal correlation time $\tau(\omega \tau \approx 1$ for the center of the transition) of $150 \mathrm{ps}<\tau<3 \mathrm{~ns}$. Without knowing where the $R_{\mathrm{NOE}} \gamma_{\mathrm{H}} / R_{1} \gamma_{\mathrm{N}}$ ratio flattens out, however, the frequency of the motion cannot be determined with great precision. 
Model-free analysis to characterize the dynamics of SARSN

While dispersion patterns may imply gross features of the dynamics, a detailed characterization of protein motions requires a more complete survey of relaxation data and interpretation using motional models. For this goal, a complete set of high-field $R_{1}$ (Fig. 2b), NOE (Fig. 3) and $R_{2}$ (Fig. 4) data was acquired and used to determine modelfree parameters for all residues of SARSN (Fig. 5). All residues were fit to a local dynamics model involving either a single order parameter $\left(S^{2}\right)$ and internal correlation time $\left(\tau_{\mathrm{e}}\right)$ (model 2, or simple model-free) (Lipari and Szabo 1982) or two order parameters $\left(S_{\mathrm{f}}^{2}\right.$ and $\left.S_{\mathrm{s}}^{2}\right)$ and internal correlation times $\left(\tau_{\mathrm{s}}\right.$ and $\tau_{\mathrm{f}}$ ), where $\tau_{\mathrm{f}}$ is assumed to be $\sim 0$ (model 5, or extended model-free) (Clore et al. 1990).

Models including an $R_{\mathrm{EX}}$ term were not selected by information criteria for any residues. This is in agreement with $R_{2}$ relaxation-dispersion experiments performed on SARSN, which also do not identify any significant $\mu$ s-ms fluctuations (data not shown). The pronounced decrease in $R_{2}$ values in the loop and $\beta$-hairpin regions (Fig. 5) is consistent with significant ps-ns motions, but not slower motions.

While the majority of residues have high order parameters and short internal correlation times typical for a stable, folded structure, residues with the above described unusual $R_{1}$ and NOE dispersions fit to model 5. These model 5 residues are characterized by high $S_{\mathrm{f}}^{2}$ and low $S_{\mathrm{s}}^{2}$ values, indicative of local order combined with largeramplitude slower fluctuations. Critically, the field dependence of the $R_{1}$ and NOE data enabled us to robustly determine the correlation time $\tau_{\mathrm{s}}$ of these slower fluctuations. The striking similarity of $\tau_{\mathrm{s}}$ of about $0.8 \mathrm{~ns}$ for the

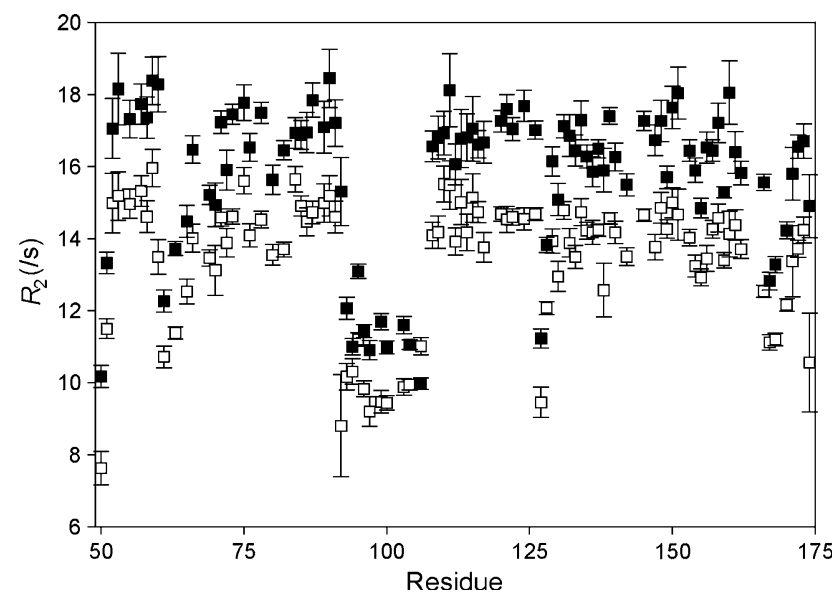

Fig. $4 R_{2}$ data show no exchange on the us-ms timescale but support fast time-scale motions in the hairpin. $R_{2}$ data are shown at ${ }^{15} \mathrm{~N}$ Larmor frequencies of $50.7 \mathrm{MHz}$ (open square), and $60.8 \mathrm{MHz}$ (filled square)
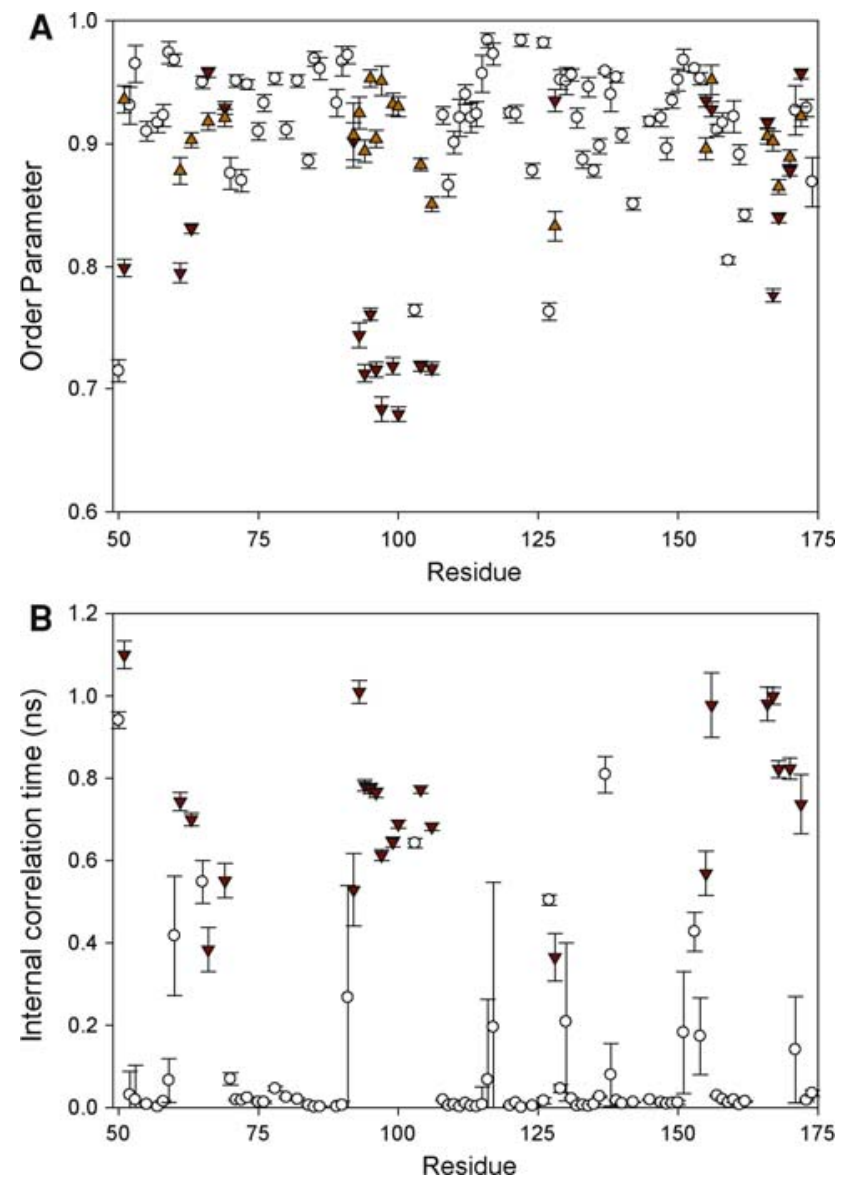

Fig. 5 Model-free parameters derived from fits of all relaxation data acquired at $500 \mathrm{MHz}$ and above fit to various model-free models (Lipari and Szabo 1982; Clore et al. 1990). a Order parameters $S^{2}$ (open circle), $S_{\mathrm{s}}^{2}$ (red filled inverted triangle), $S_{\mathrm{f}}^{2}$ (orange filled triangle). $\mathbf{b}$ Corresponding internal correlation times $\tau_{\mathrm{e}}$ (open circle) or $\tau_{\mathrm{s}}$ (red filled inverted triangle). Error bars are derived from 150 Monte Carlo simulations

entire $\beta$-hairpin and adjacent loops (Figs. 1, 5) suggests that these residues undergo a correlated motion as a stable structural unit. This is consistent with the structure showing a regular hydrogen-bonded $\beta$-hairpin as well the chemical shifts in this region (Cornilescu et al. 1999).

The high accuracy of the determined $\tau_{\mathrm{s}}$ values arises from the crossover in the $R_{1}$ data and the pronounced NOE dispersion data as illustrated by simulated relaxation parameters (Fig. 6). These theoretical curves emphasize the value of field-cycling data in identifying the $R_{1}$ crossover and thereby defining the timescale of the collective mesodynamics.

We want to note that although the observed relaxation rates at 17.3 and $30.2 \mathrm{MHz}$, obtained by field-cycling, qualitatively agree with high-field observations, the lowfield $R_{1}$ values are 45 and $30 \%$ lower than predicted from model-free parameters using high-field data, respectively. Error from interference between CSA and dipolar relaxation 

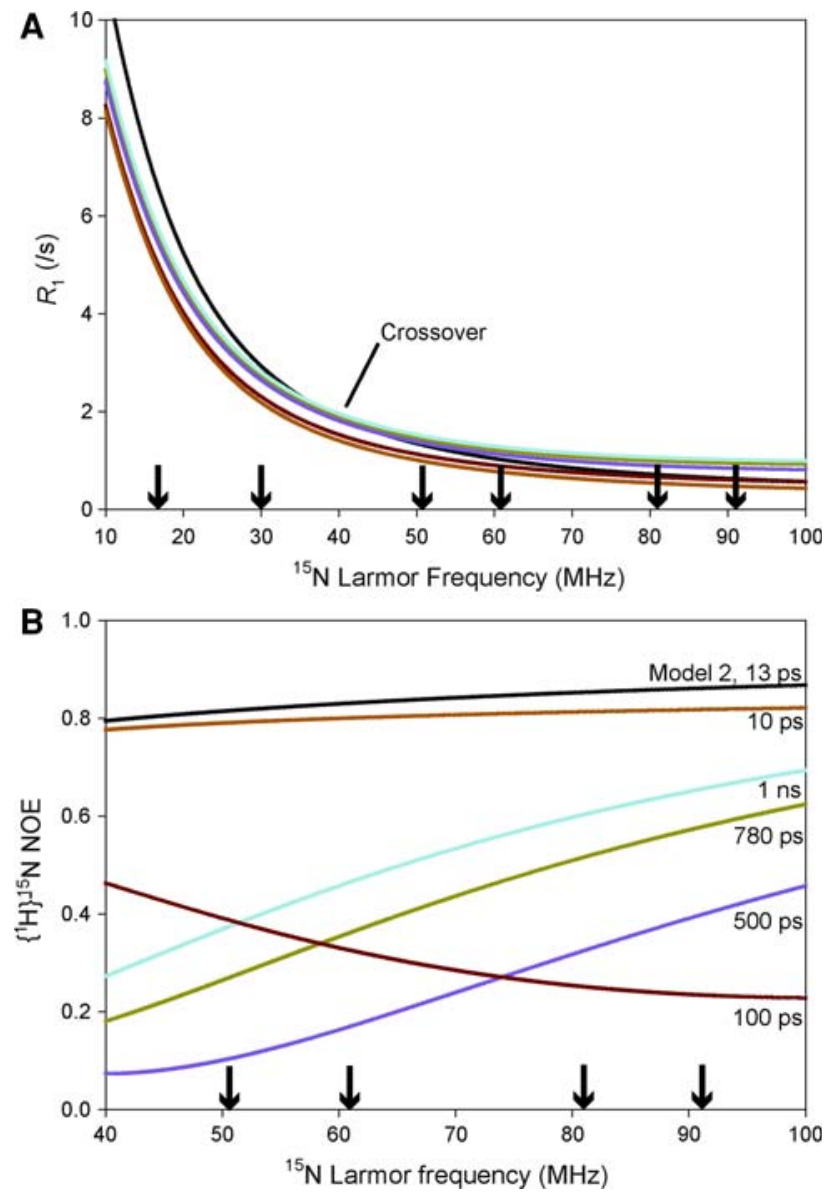

Fig. 6 Illustration of crossover behavior of $R_{1}$ rates and $\left\{{ }^{1} \mathrm{H}\right\}-{ }^{15} \mathrm{~N}$ NOE field-dependence due to the timescale of internal motions. Relaxation rates (Abragam 1961) are predicted from spectral densities calculated from model-free parameters for a protein with a global correlation time of $11.9 \mathrm{~ns}$, the value for our system. a $R_{1}$ values predicted from model 2 for $S^{2}=0.85, \tau_{\mathrm{e}}=13 \mathrm{ps}$ (black line), and model 5 for $S_{\mathrm{f}}^{2}=0.89, S_{\mathrm{s}}^{2}=0.71$, and $\tau_{\mathrm{s}}=10 \mathrm{ps}$ (orange line), $\tau_{\mathrm{s}}=100 \mathrm{ps}($ red line $), \tau_{\mathrm{s}}=500 \mathrm{ps}($ purple line $), \tau_{\mathrm{s}}=780 \mathrm{ps}($ green line), and $\tau_{\mathrm{s}}=1.0 \mathrm{~ns}$ (cyan line). The black and green lines correspond to actual fitted parameters for residues 142 (in the stable globular part of SARSN) and 94 (in the $\beta$-hairpin with complex motions), respectively. Note the crossover point for internal correlation times in the $0.5-1 \mathrm{~ns}$ range. b Predicted steady-state $\left\{{ }^{1} \mathrm{H}\right\}-{ }^{15} \mathrm{~N}$ NOE values (same scheme). Note the strong increase in the NOE value with increasing field for internal correlation times in the $0.5-1 \mathrm{~ns}$ range. Arrows indicate fields used in this study

mechanisms is of particular concern here, due to the inability to regularly invert proton polarization during the relaxation period in the field-cycled experiments. While this effect certainly contributes to the observed bias towards too low $R_{1}$ values, it is not possible to account for the entire effect at 17.3 and $30.2 \mathrm{MHz}$ using analytical calculations of $\eta_{\mathrm{z}}$, implying that the observed bias cannot be attributed to any single source of interference.

Additionally, in the fringe field of a magnet, the field strength decreases constantly with increasing distance from the center of the coil. As a result, the field is inhomogeneous over the $1.5 \mathrm{~cm}$ length of the sample, with a maximal variation of $1.4 \mathrm{~T}$ at $30.2 \mathrm{MHz}(7 \mathrm{~T})$. The remaining difference between prediction and experiment could in part result from this field inhomogeneity, but could also arise from technical error or from some previously unrecognized shortcoming of the model-free theory. These questions should be investigated further in future by a more complete survey between 5 and $9 \mathrm{~T}$, or possibly at lower field using ${ }^{13} \mathrm{C}$ carbonyl relaxation. Below $4 \mathrm{~T}$ the relaxation rate is expected to be too fast to be measured with our shuttle device for any nuclear spin adjacent to a geminal proton because of relaxation of the measured nuclear spin by the proton. For the results presented here, this uncertainty does not significantly affect the conclusions.

\section{MD simulations of SARSN in explicit solvent}

Although the similarity in correlation times for the entire $\beta$ hairpin and a number of residues that are far away in sequence but close in structure suggests a collective motion, the ensemble nature of NMR measurements precludes a direct verification. For this goal, we performed a $15 \mathrm{~ns}$ all-atom MD simulation of the SARSN N-terminal domain in explicit solvent. Here, correlated motions can be directly identified, since the simulations are performed on a single molecule.

Consistent with the high order parameters fitted for the majority of the domain, the globular part of the protein is stable, without detectable structural drift (Fig. 7a, b). The $\beta$-hairpin is also internally stable-although it is exposed to solvent, it does not show signs of partial unfolding during the simulation. When the hairpin is superimposed for all snapshots from the simulation, the fluctuations remain within $2 \AA$ for the $\beta$-hairpin throughout the trajectory (Fig. 7b). However, the simulations show a large amplitude motion of the $\beta$-hairpin as a rigid body relative to the globular portion of the protein (Fig. 7a, b). The low internal fluctuation within the $\beta$-hairpin is in agreement with high $S_{\mathrm{f}}^{2}$, while the large displacement of the $\beta$-hairpin corresponds to the low $S_{\mathrm{s}}^{2}$ values calculated form the NMR experiments. Moreover, the timescale of the $\beta$-hairpin displacement is in the ns time regime, in agreement with the $\tau_{\mathrm{s}}$ of $0.8 \mathrm{~ns}$ from the NMR experiments.

In addition to extensive mesodynamics in the $\beta$-hairpin, the NMR relaxation data revealed motions with similar correlation times for loops encompassing residues 60-64 and 127-130. The MD simulations now provide a rationale for this observation: during the simulations, direct and water-mediated hydrogen bonds form between several residues in the hairpin and these flexible loops. For example, the side-chain of R90 hydrogen-bonds temporarily to residues in both loops (Fig. 7c). These interactions seem to be responsible for coordinating motions of the 


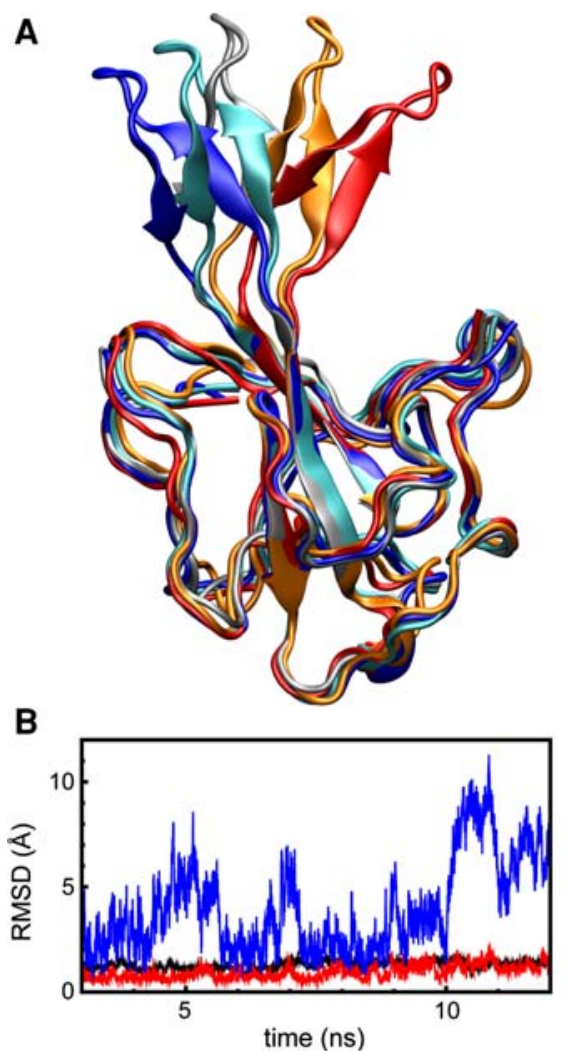

Fig. 7 The $\beta$-hairpin moves collectively as a rigid body and its motion is correlated to motions in adjacent loops. a Representative snapshots from the $15 \mathrm{~ns}$ MD trajectory in explicit solvent. The structures are aligned on the globular domain. Trajectory snapshots are blue $(5,340 \mathrm{ps})$ cyan $(4,853 \mathrm{ps})$, orange $(10,271 \mathrm{ps})$, and red $(11,033 \mathrm{ps})$; the original X-ray structure (PDB code: $2 \mathrm{OFZ})$ is in gray. b Time traces of RMSD with respect to the crystal structure. RMSD of the globular portion of the domain when the protein is aligned on the globular domain (as in a) is shown in black, and the RMSD of the hairpin in blue. The RMSD of the hairpin when the

hairpin and those loops, explaining the similarity of relaxation dispersions at these sites. Strikingly, the MD simulations elucidate the collective nature of the mesodynamics of the entire $\beta$-hairpin and the correlation of motions between the hairpin and the 60-64 loop. These correlations can be computed from the time traces of atomic fluctuations within one molecule through a covariance matrix (Fig. 7d), emphasizing the value of MD simulations to complement the NMR experiments.

\section{Conclusions}

The advantages of examining relaxation properties of proteins at multiple field strengths are well-known, and due to the limitations of resolution and configuration, unidirectional expansion of measurements to higher fields has been the natural progression. Here we have expanded our sampling of the spectral density in both directions using a
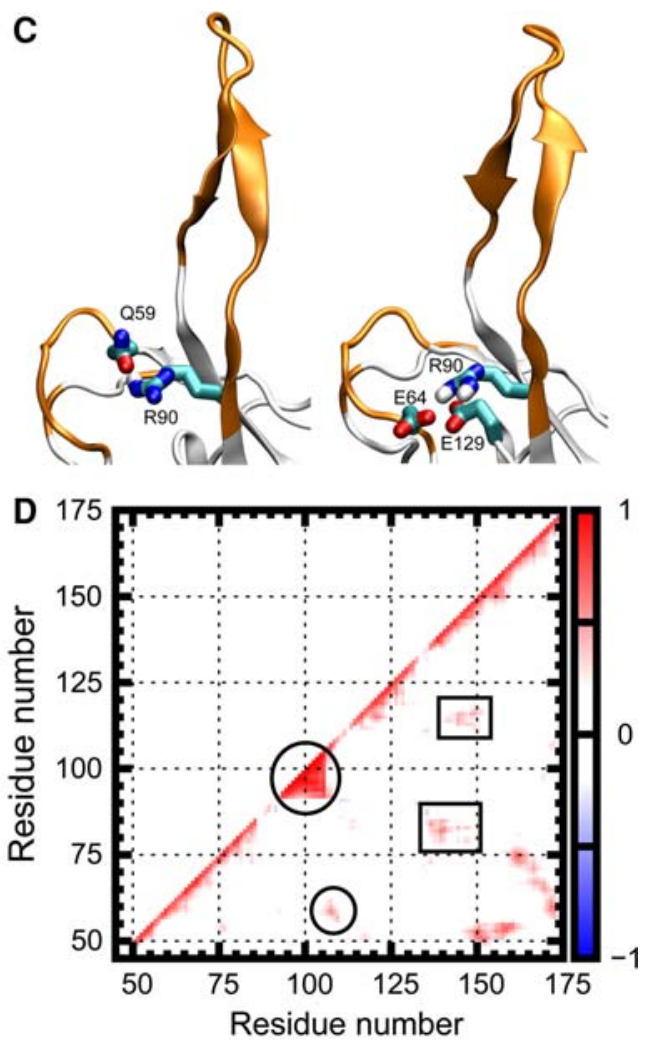

protein is aligned on the hairpin is shown in red. c Representative snapshots depicting hydrogen bonds between the side chain of R90 and backbone and side-chain moieties in the 60-64 loop and E129. d Plot showing correlated (red) and anti-correlated (blue) motions of SARSN over the duration of a 15 ns trajectory in a covariance matrix (Ichiye and Karplus 1991). Motions within the $\beta$-hairpin and between the $\beta$-hairpin with the 60-64 loop are correlated (marked by circles), in addition to correlated motions between the $\beta$-strands in the core of the protein (marked by boxes)

field-cycling apparatus and high-field magnets to measure $R_{1}$ relaxation in a heterogeneously dynamic domain from the SARS coronavirus at atomic resolution.

Besides the proof of principle that site-specific relaxation rates can be determined at low field while retaining much of the sensitivity and all of the resolution of a highfield spectrometer, the experiments allowed a precise determination of the internal correlation times of residues with complex motional models (model 5). Given that these residues have very similar internal correlation times and cluster structurally together, we suggest that the relaxation behavior is the result of correlated motions of the hairpin and the 60-64 loop. This model is buttressed by MD simulations. The $\beta$-hairpin and the 60-64 loop coincide with several previously-identified sites where SARSN engages in protein-protein and protein-nucleic acid interactions, including a ubiquitination site at K62. That areas of significant flexibility in SARSN coincide with residues important for binding interactions is not surprising, because 
disordered regions in proteins are often associated with promiscuous binding activity (Fink 2005). However our current data do not provide mechanistic insight into the relation between dynamics and SARS function.

Although the technique of measuring relaxation in the fringe field limits the accuracy of the relaxation rates, the data from low-field experiments are qualitatively consistent with the findings from high-field and ultra-high-field spectrometry. With further refinement of equipment and technique it should be possible to improve the sensitivity and accuracy of these measurements substantially. The methods of field-cycling (Roberts et al. 2004; Sivanandam et al. 2009) will continue to be very useful in other contexts: for example, experiments with ${ }^{13} \mathrm{C}$ carbonyls where the absence of an attached proton will probably make measurements to zero field feasible, as they are for ${ }^{31} \mathrm{P}$.

Acknowledgments The authors would like to thank Geoffrey Armstrong of the Rocky Mountain Regional NMR facility for assistance in acquiring the $900 \mathrm{MHz}$ spectra, and Karl Koshlap of the University of North Carolina School of Pharmacy for assistance in acquiring high-field data at $500 \mathrm{MHz}$. This work was in part supported by the Howard Hughes Medical Institute, NIH grants to D.K. (GM 67963 \& GM 062117) and A.R (GM 077974), a DOE grant to D.K (FG02-05ER15699), and instrumentation grants from the NSF and the Keck foundation to D.K.

Open Access This article is distributed under the terms of the Creative Commons Attribution Noncommercial License which permits any noncommercial use, distribution, and reproduction in any medium, provided the original author(s) and source are credited.

\section{References}

Abragam A (1961) The principles of nuclear magnetism. Oxford, Clarendon Press

Brooks CL, Karplus M (1983) Deformable stochastic boundaries in molecular-dynamics. J Chem Phys 79:6312-6325

Brooks BR, Bruccoleri RE et al (1983) CHARMM-a program for macromolecular energy, minimization, and dynamics calculations. J Comput Chem 4:187-217

Brunger A, Brooks CL, Karplus M (1984) Stochastic boundaryconditions for molecular-dynamics simulations of St2 water. Chem Phys Lett 105:495-500

Clarkson MW, Gilmore SA, Edgell MH, Lee AL (2006) Dynamic coupling and allosteric behavior in a nonallosteric protein. Biochemistry 45:7693-7699. doi:10.1021/Bi0606521

Clore GM, Szabo A et al (1990) Deviations from the simple 2-parameter model-free approach to the interpretation of $\mathrm{N}-15$ nuclear magnetic-relaxation of proteins. J Am Chem Soc 112:4989-4991

Cornilescu G, Delaglio F, Bax A (1999) Protein backbone angle restraints from searching a database for chemical shift and sequence homology. J Biomol NMR 13:289-302

d'Auvergne EJ, Gooley PR (2003) The use of model selection in the model-free analysis of protein dynamics. J Biomol NMR 25:25-39

Delaglio F, Grzesiek S et al (1995) NMRPipe-a multidimensional spectral processing system based on Unix pipes. J Biomol NMR 6:277-293
Farrow NA, Muhandiram R et al (1994) Backbone dynamics of a free and a phosphopeptide-complexed Src homology-2 domain studied by N-15 NMR relaxation. Biochemistry 33:5984-6003

Fink AL (2005) Natively unfolded proteins. Curr Opin Struct Biol 15:35-41. doi:10.1016/j.sbi.2005.01.002

Grubmuller H (1995) Predicting slow structural transitions in macromolecular systems-conformational flooding. Phys Rev E 52:2893-2906

Hsieh PK, Chang SC et al (2005) Assembly of severe acute respiratory syndrome coronavirus RNA packaging signal into virus-like particles is nucleocapsid dependent. J Virol 79: 13848-13855. doi:10.1128/Jvi.79.22.13848-13855.2005

Huang QL, Yu LP et al (2004) Structure of the N-terminal RNAbinding domain of the SARS $\mathrm{CoV}$ nucleocapsid protein. Biochemistry 43:6059-6063. doi:10.1021/Bi036155b

Ichiye T, Karplus M (1991) Collective motions in proteins-a covariance analysis of atomic fluctuations in molecular-dynamics and normal mode simulations. Proteins 11:205-217

Johnson BA, Blevins RA (1994) NMRView-a computer-program for the visualization and analysis of NMR data. J Biomol NMR 4:603-614

Kimmich R (1996) Molecular motions: $T_{1}$ frequency dispersion in biological systems. In: Grant DM, Harris RK (eds) Encyclopedia of nuclear magnetic resonance, vol 5. Wiley, Hoboken, NJ, pp 3083-3088

Lee AL, Flynn PF, Wand AJ (1999) Comparison of H-2 and C-13 NMR relaxation techniques for the study of protein methyl group dynamics in solution. J Am Chem Soc 121:2891-2902

Lipari G, Szabo A (1982) Model-free approach to the interpretation of nuclear magnetic-resonance relaxation in macromolecules. 1. Theory and range of validity. J Am Chem Soc 104:4546-4559

Luchinat C, Parigi G (2007) Collective relaxation of protein protons at very low magnetic field: a new window on protein dynamics and aggregation. J Am Chem Soc 129:1055-1064. doi:10.1021/ $\mathrm{Ja} 0633417$

Luo H, Ye F et al (2004) In vitro biochemical and thermodynamic characterization of nucleocapsid protein of SARS. Biophys Chem 112:15-25. doi:10.1016/j.bpc.2004.06.008

MacKerell AD, Bashford D et al (1998) All-atom empirical potential for molecular modeling and dynamics studies of proteins. J Phys Chem B 102:3586-3616

Mackerell AD, Feig M, Brooks CL (2004) Extending the treatment of backbone energetics in protein force fields: limitations of gasphase quantum mechanics in reproducing protein conformational distributions in molecular dynamics simulations. J Comput Chem 25:1400-1415. doi:10.1002/Jcc.20065

Redfield AG (2003) Shuttling device for high-resolution measurements of relaxation and related phenomena in solution at low field, using a shared commercial $500 \mathrm{MHz}$ NMR instrument. Magn Reson Chem 41:753-768. doi:10.1002/Mrc.1264

Roberts MF, Cui QZ et al (2004) High-resolution field-cycling NMR studies of a DNA octamer as a probe of phosphodiester dynamics and comparison with computer simulation. Biochemistry 43:3637-3650. doi:10.1021/Bi035979q

Saikatendu KS, Joseph JS et al (2007) Ribonucleocapsid formation of severe acute respiratory syndrome coronavirus through molecular action of the $\mathrm{N}$-terminal domain of $\mathrm{N}$ protein. $\mathrm{J}$ Virol 81:3913-3921. doi:10.1128/Jvi.02236-06

Sivanandam VN, Cai JF, Redfield AG, Roberts MF (2009) Phosphatidylcholine "Wobble" in vesicles assessed by high-resolution C-13 field cycling NMR spectroscopy. J Am Chem Soc 131:3420-3421. doi:10.1021/Ja808431h

Surjit M, Lal SK (2008) The SARS-CoV nucleocapsid protein: a protein with multifarious activities. Infect Genet Evol 8:397405. doi:10.1016/j.meegid.2007.07.004 
Surjit M, Liu BP, Chow VTK, Lal SK (2006) The nucleocapsid protein of severe acute respiratory syndrome-coronavirus inhibits the activity of cyclin-cyclin-dependent kinase complex and blocks $\mathrm{S}$ phase progression in mammalian cells. J Biol Chem 281:10669-10681. doi:10.1074/jbc.M509233200

Tan YW, Fang SG et al (2006) Amino acid residues critical for RNAbinding in the N-terminal domain of the nucleocapsid protein are essential determinants for the infectivity of coronavirus in cultured cells. Nucleic Acids Res 34:4816-4825. doi:10.1093/ Nar/Gkl650
Vuister GW, Wang AC, Bax A (1993) Measurement of 3-bond nitrogen carbon-J couplings in proteins uniformly Enriched in $\mathrm{N}-15$ and C-13. J Am Chem Soc 115:5334-5335

Zhang X, Wu KL et al (2007) Nucleocapsid protein of SARS-CoV activates interleukin-6 expression through cellular transcription factor NF-kappa B. Virology 365:324-335. doi:10.1016/j.virol. 2007.04.009

Zuniga S, Sola I et al (2007) Coronavirus nucleocapsid protein is an RNA chaperone. Virology 357:215-227. doi:10.1016/j.virol. 2006.07.046 